\title{
European DEMO first wall shaping and limiters design and analysis status
}

\author{
Z. Vizvary ${ }^{a^{*}}$, W. Arter ${ }^{\mathrm{a}}$, C. Bachmann ${ }^{\mathrm{c}}$, T. R. Barrett ${ }^{\mathrm{a}}$, B. Chuilon ${ }^{\mathrm{a}}$, P. Cooper ${ }^{\mathrm{a}}$, E. Flynn $^{\mathrm{a}}$, \\ M. Firdaouss ${ }^{b}$, T. Franke ${ }^{\text {c,d }}$, J. Gerardin ${ }^{b}$, R. Gowland ${ }^{a}$, M. Kovari ${ }^{a}$, F. Maviglia ${ }^{c}$, M. L. Richiusa ${ }^{a}$, \\ E. V. Rosa Adame ${ }^{\mathrm{a}}$, C. Vorpahl ${ }^{\mathrm{c}}$, A. Wilde ${ }^{\mathrm{a}}$, Y. Xue ${ }^{\mathrm{a}}$ \\ ${ }^{a} U K A E A$, Culham Science Centre, Abingdon, Oxon, OX14 3DB, UK \\ ${ }^{b}$ IRFM CEA Cadarache, 13108, Saint Paul lez Durance, France \\ ${ }^{c}$ EUROfusion - Programme Management Unit, Boltzmannstrasse 2, 85748 Garching, Germany \\ ${ }^{d}$ Max-Planck-Institut für Plasmaphysik, Garching, Germany
}

\begin{abstract}
The anticipated heat flux limit of the European DEMO first wall is $\sim 1-2 \mathrm{MW} / \mathrm{m}^{2}$. During transient and off normal events, the heat load deposited on the wall would be much larger than the steady state heat load and exceed the first wall limit, therefore the breeding blanket first wall needs to be protected. This involves dedicated discrete limiters in certain regions of the machine that would take the brunt of the heat load as well as adequate shaping of the first wall. The current concept envisages limiters at a few (3-4) equatorial ports to cope with the ramp-up of the plasma; upper limiters (in $\sim 8$ upper ports) are considered for upward vertical displacement events. Two design options have been considered for these limiters: a modular design where the limiter plasma facing components are attached to individual plates that are assembled together so that transient electromagnetic loads can be reduced, and in case of damage the plates can be replaced/repaired individually; and a divertor-like design where the plasma facing components are attached to a single Eurofer cassette. Other limiters considered include inner wall limiters in case of plasma contraction and lower limiters may be needed for downward vertical displacement events. The thermal hydraulic finite element analysis results show that the integrity of the cooling pipes can be maintained during the anticipated transient events. The limiters are considered to be sacrificial and designed to be replaceable independently from the breeding blanket system. The design has to allow that installation, removal or replacement of the limiters can be performed remotely. Strategy to tackle outstanding issues and required R\&D is also discussed.
\end{abstract}

Keywords: DEMO, First Wall shaping, Limiter, PFC, thermo-hydraulic analysis

\section{Introduction}

The EU DEMO breeding blanket (BB) first wall (FW) is capable of withstanding heat fluxes in the $\sim 1-2 \mathrm{MW} / \mathrm{m}^{2}$ [1]. The limited capability is caused by the relatively low thermal conductivity of the heat sink material Eurofer. Analyses so far show that this limit can be respected during normal operation [2-3]. However, in case of normal and off normal transients the heat flux on the FW can exceed well the above-mentioned limit. The DEMO key design integration issue - 1 (KDII-1) is concerned with the performance and feasibility of limiters during these transients [4-5]. The envisaged worst plasma transients are:

- the ramp-up, which happens regularly at every single pulse;

- $\quad$ upward vertical displacement event (VDE);

- downward VDE;

- H-L transition (loss of confinement).

This paper focuses on the latest development in the engineering solutions to deal with these events. The physics behind the above transient scenarios in the EU DEMO is detailed in [6].
In order to protect the $\mathrm{BB} F W$ various limiters are proposed that can withstand the transient heat loads, or at least while providing protection to the $\mathrm{BB} F W$ the structural integrity of its heat sink structure can be maintained.

The listed transient events affect different locations in the plasma chamber and therefore each event has its own dedicated limiter.

The naming convention of the limiters and the transient events they are designed for are in Table 1 . The locations for the limiter are also shown on the schematic view (Figure 1).

Table 1: Transients and limiters
\begin{tabular}{|l|c|l|c|}
\hline Transient event & $\begin{array}{l}\text { Number } \\
\text { on Figure } \\
1\end{array}$ & Limiter & $\begin{array}{l}\text { Number of } \\
\text { limiters }\end{array}$ \\
\hline Ramp-up & 1 & $\begin{array}{l}\text { Outboard Mid-plane } \\
\text { Limiter (OML) }\end{array}$ & $(3-) 4$ \\
\hline Upward VDE & 2 & Upper Limiter (UL) & 8 \\
\hline $\begin{array}{l}\text { Downward } \\
\text { VDE }\end{array}$ & 4 & $\begin{array}{l}\text { Outboard Lower } \\
\text { Limiter (OLL) }\end{array}$ & $(3-) 4$ \\
\hline H-L transition & 3 & $\begin{array}{l}\text { Inboard Mid-plane } \\
\text { Limiter (IML) }\end{array}$ & $(3-) 4$ \\
\hline
\end{tabular}


Important to note is that the limiters are not toroidally continuous components, the envisaged number of each limiter is also included in Table 1. The OML, OLL and IML would be placed in between two breeding blanket segments so that both segments would require a cut-out about half of the segment width to avoid having to split them into two. This will affect their structural integrity of the blanket segments. The impact of the cut-outs is not assessed within the limiter design tasks. The limiters also reduce the available breeding blanket surface area and thus have an impact on the tritium breeding ratio.

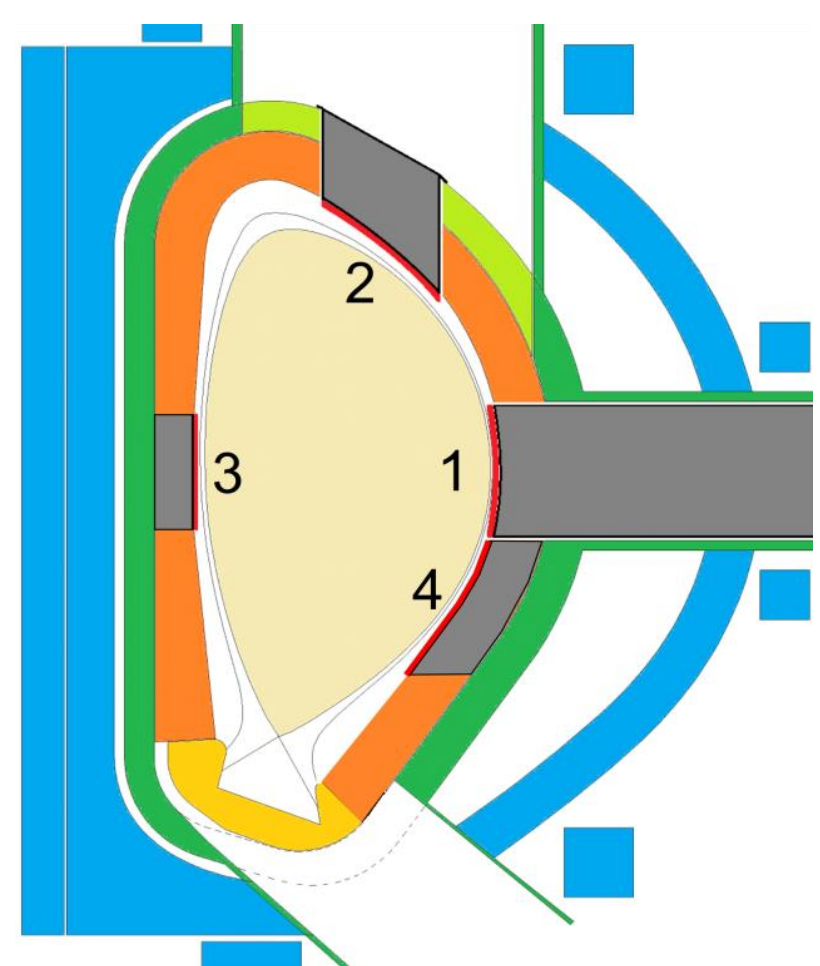

Figure 1: Schematic view of the EU DEMO indicating the positions which the limiters occupy.

The work presented here summarises the latest status of the limiter development for each of the limiters and identifies the design focus for the short-term future.

\section{Limiter PFC concept}

Although their function is different the technology of the limiter plasma-facing components (PFCs) can be similar to the ITER and DEMO divertor technology [7-9]. Both the divertor and limiter components are designed to withstand high heat flux, however unlike the divertor which has to withstand a high steady-state heat flux, the heat flux for the limiters can be extremely high at the same time the duration of these loads are short (Table 2). In fact, the thermal loads can be so large that the plasma facing tungsten armour can be damaged (i.e.: molten, evaporated, cracked). Therefore, frequent replacement may be required.
Table 2: Typical heat loads for limiters

\begin{tabular}{|l|l|l|}
\hline Limiter & $\begin{array}{l}\text { Design heat } \\
\text { load }\end{array}$ & Duration \\
\hline OML & $5-10 \mathrm{MW} / \mathrm{m}^{2}$ & $20-60 \mathrm{~s}$ \\
\hline UL & $\sim 25 \mathrm{GW} / \mathrm{m}^{2}$ & $\sim 4 \mathrm{~ms}$ \\
\hline OLL & $\sim 150 \mathrm{GW} / \mathrm{m}^{2}$ & $\sim 4 \mathrm{~ms}$ \\
\hline IML & $10-20 \mathrm{MW} / \mathrm{m}^{2}$ & $5 \mathrm{~s}$ \\
\hline
\end{tabular}

The main candidates are tungsten monoblock PFCs that are cooled by $\mathrm{CuCrZr}$ heat sink. The armour will be thick [6] to limit the heat conducted to the heat sink during the transients. According to our current knowledge the $\mathrm{CuCrZr}$ heat sink is likely to have an irradiation lifetime (possibly around 1-2 full power years (fpy) [11]) that is lower than the plant life ( 6fpy). This also indicates that the limiters will have to be replaced more frequently than the BB segments and therefore have to be designed so that they can be replaced independently.

Various PFC technologies are considered within the divertor work package [10]: ITER-like; thermal break; composite; chromium; functionally graded; W laminate; water jet and even He cooled.

One of these is described here, that belongs to the thermal break category. The concept PFC features a Eurofer strip as thermal break (Figure 2) to move the heat around (to delay its transit to the coolant) and thus reduce the maximum pipe/pipe-bore temperatures.

\section{Plasma facing side}

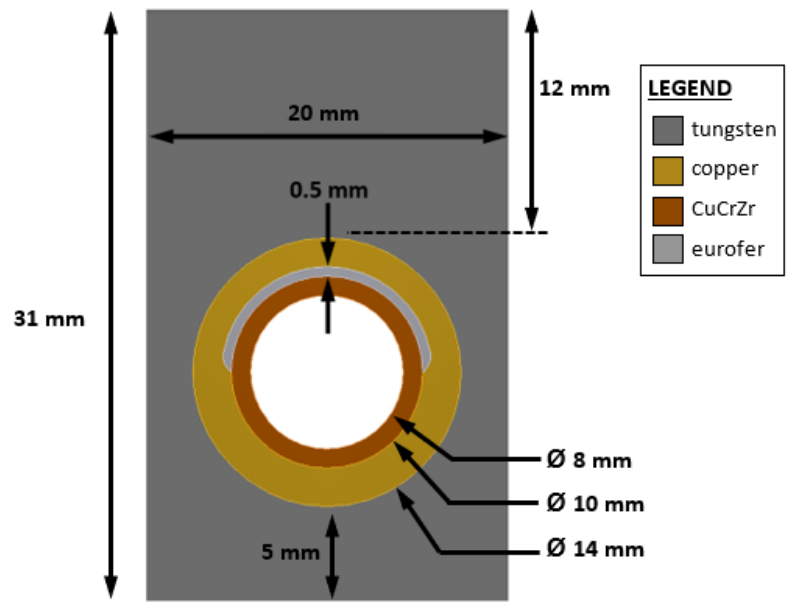

Figure 2: Monoblock cross-section for upper limiter.

Previously a wide monoblock with complex cuts has had been proposed [12]; recently it was reversed to a simpler monoblock (Figure 2) which is easier to manufacture and allows more flexibility in geometry. This is however at the price of having more cooling pipes in the vessel as the monoblock is narrower to achieve similar capability. 


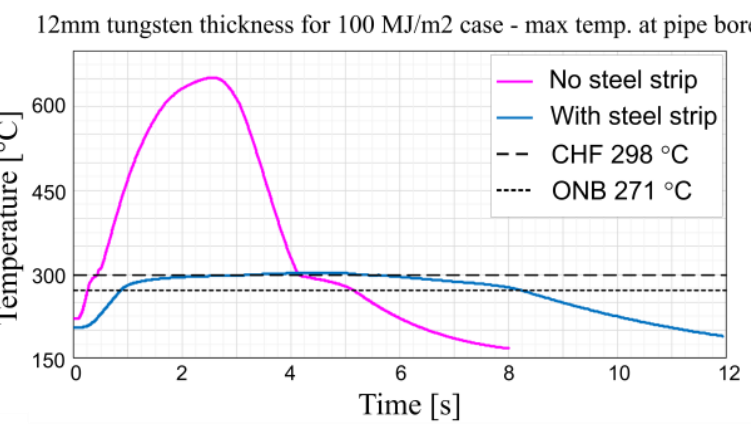

Figure 3: Comparison of pipe bore peak temperature with and without thermal break for $12 \mathrm{~mm}$ tungsten monoblock thickness.

Analyses have been done considering the typical flow boiling curve, and formulae such as the Sieder and Tate correlation for subcooled conditions, modified to include a twisted tape [13], Bergles and Rohsenov correlations for the onset of nucleate boiling [13], correlations from Thom (modified by CEA) to compute the nucleate boiling flux and HTC [14] and a modified Tong correlation proposed by Schlosser for estimating the critical heat flux [14]. The results show that using the thermal break the $\mathrm{CuCrZr}$ pipe bore temperature stays in the region of $\sim 300{ }^{\circ} \mathrm{C}$ and there is only a short period of time when the wall critical heat flux (CHF) is slightly exceeded (Figure 3, Upper Limiter analysis). Increasing the tungsten thickness could reduce the pipe bore temperature. Analysis suggest that the tungsten thickness could be increased to $25 \mathrm{~mm}$ without reaching the recrystallisation temperature at steady state conditions.

As mentioned above, the current concept is based on the ITER and DEMO divertor technology, but alternative options could also be explored both regarding the design (i.e.: hypervapotron heat sink) and the material composition. Alternative PFC technologies based on porous and additive manufactured tungsten are also investigated within the divertor work package.

\section{Limiter design options}

Two design options have been considered in the development of the limiters. In both cases the PFCs are attached to a shield plug: a water-cooled Eurofer steel structure. The shield plug is not just a structural part to which the PFCs are attached to, but they also have to provide sufficient shielding to the structures (port plug, vacuum vessel) behind.

\subsection{Plate-based design}

The "plate-based" design follows the principle of the once proposed ITER port limiter [15] or the JET ITER-like wall tile design [16], where the components contain "cuts" so that the transient electromagnetic loads (eddy currents) can be kept as low as possible. The "cuts" are designed to prevent the possibilities of large current loops induced in the components during plasma disruptions. If the cuts run poloidally the poloidal current loops can be prevented, while dividing the poloidal extent into smaller section current loops due to toroidal field change (in the thermal quench phase, while the plasma is doing a poloidal inward shift due to shrinkage by loss of energy) can be reduced. Analyses so far confirmed that indeed the eddy currents (especially during the current quench) can be reduced this way [17].

Such shield block can be realised by manufacturing distinct Eurofer plates which are assembled together by attaching them to a back frame to form the shield plug. The plates would be relatively easy to manufacture and would be used several hundred times in the whole reactor, with variants for each limiter (although this solution may not be viable for the IML). This modular build would also offer flexibility and smaller component size that builds up the limiter. The modularity could potentially allow the repair or replacement of damaged monoblocks/monoblock sections, although handling an irradiated limiter at these sizes certainly would not be easy to maintain remotely.

The actual plates could be made from two parts using hot isostatic pressing (HIP). This would also allow bespoke internal cooling channel geometry inside the plates. This can be optimized for both to the desired plate temperature and shielding capability.

More work needs to be done on the attachment of these plates to make it a viable option. Estimated halo current loads could be large (especially for the UL and OLL). Attaching the plates only in the back would challenge any attachment system. Attaching the plates together (for example by preloaded tie-rods) could increase the strength. This would have to be done via a high resistivity path, to avoid compromising the resilience against eddy currents. Concerns regarding the loss of preload due to irradiation and high operating temperature of the uncooled tie-rods in the proximity of the plasma require novel solutions. Work is ongoing to understand how the irradiation induced stress relaxation and creep in such assembly could be managed.

\subsection{Divertor-like design}

The divertor-like design is building on the information already learned from the divertor development [16]. As said the technology will be very similar, even the coolant conditions are to be shared $\left(180{ }^{\circ} \mathrm{C} / 130{ }^{\circ} \mathrm{C}\right.$ water, $3.5 \mathrm{MPa} / 5 \mathrm{MPa})$ given the same material choices $(\mathrm{CuCrZr}$ and Eurofer, respectively [18-19]). Building on the divertor development could shorten the limiter development path, share resources for future R\&D and ensure the consistency among the various components.

The size and shape of the limiters are different from the divertor (and from each other), the number of PFC cooling pipes and the length of these pipes will also vary and therefore for each limiter the internal structure of the "cassette" will have to be optimised. The heat load on the cassette is driven by the volumetric heat load during normal operation due to the large volume and thus mass flow rate. The internal cooling layout is not trivial. 


\section{The Limiters}

\subsection{Outboard Mid-plane Limiter (OML)}

Among the various plasma transient events the ramp-up is the one that occurs on a regular basis. The maximum envisaged heat flux is $5-10 \mathrm{MW} / \mathrm{m}^{2}$ for tens of seconds (20-60s). During the ramp-up the plasma will touch the OML [6]. We foresee 4 of such limiters periodically placed 90 degrees apart (alternatively it is studied to use only 3 to save equatorial ports). Each limiter is attached to a port plug and integrated in an equatorial port. The port itself is toroidally offset in order to avoid splitting the blanket segments vertically (Figure 4) [20]. The overall surface area of this limiter is $1.1 \mathrm{~m} \times 2.8 \mathrm{~m}$.

During ramp-up these limiters will have to be well aligned; a mechanism is envisaged that would allow the fine adjustment of the limiter $( \pm 5 \mathrm{~mm})$ to achieve this after assembly [20].

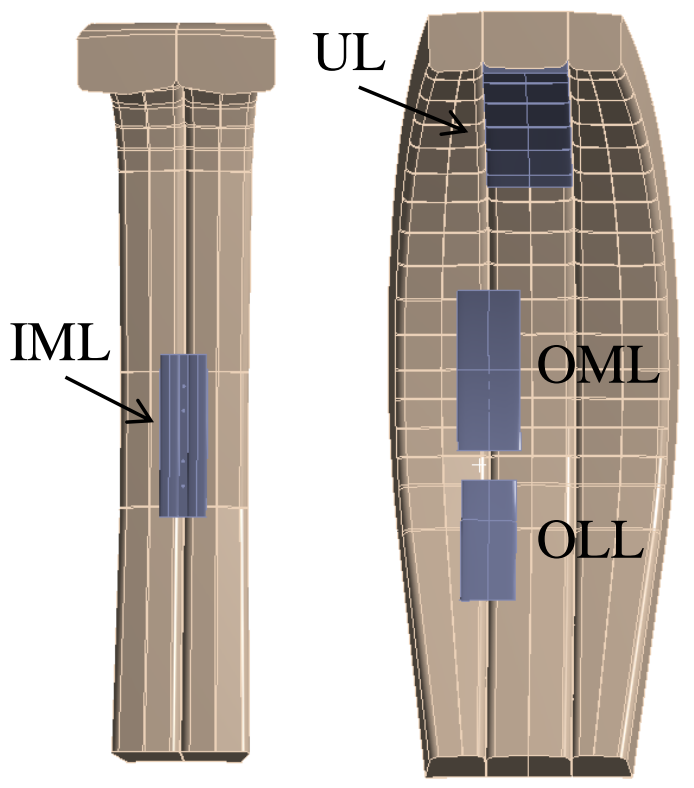

Figure 4: Limiters in a sector: left, inboard view, right, outboard view (without PFCs).

\subsection{Upper Limiter (UL)}

Avoiding disruptions and VDEs cannot be guaranteed based on present plasma scenarios. The energy released during the upward VDE is huge: $\sim 1.3$ GJ during the thermal quench in $\sim 4 \mathrm{~ms}$ [21], followed by the current quench with $\sim 1$ GJ within $\sim 250 \mathrm{~ms}$ [6]. This enormous heat load would destroy the BB FW. To prevent this every second upper port would include an UL similarly attached to the central upper port plug. The 8 ULs are replacing the top section of the central outboard blanket segments [22]. This also means that the port plug has to provide a load path towards the inboard segments.

The estimated heat load is so large for the UL that it seems to be unavoidable that the tungsten monoblocks would melt/evaporate [6]. In the design the focus is on ensuring the integrity of the heat sink structure in such event, so that the coolant cannot leak into the plasma chamber [5]. The overall surface area of this limiter is $1.5 \mathrm{~m} \times 3.4 \mathrm{~m}$.
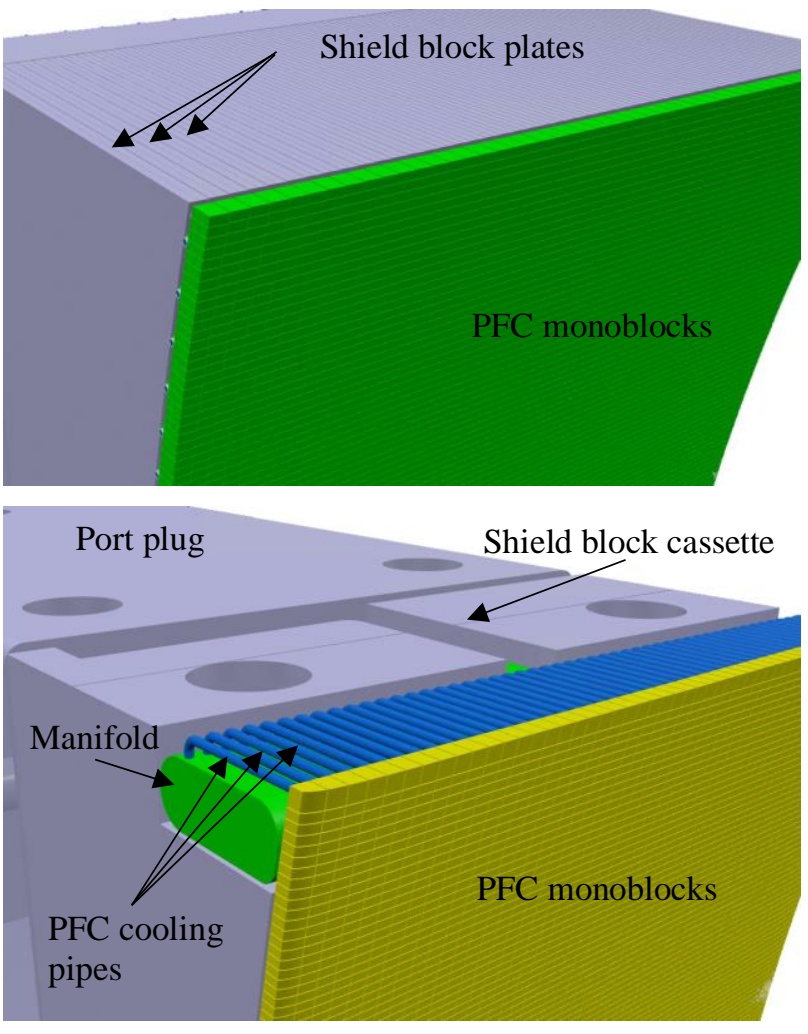

Figure 5: Upper left corner of Outboard Mid-plane Limiter: above, plate-based design below, divertor-like design.

\subsection{Outboard Lower Limiter (OLL)}

In case of a downward VDE an OLL is being considered to protect the BB. This work is in the early stages [5]. They need to be located just slightly further down from the OMLs offering an opportunity to install/remove these limiters and provide coolant connections also through the equatorial port. The OLL will need to be extended upward (compared to Figure 4) to achieve this. It also means that there will be only (3-)4 OLL, meaning even higher heat loads than on the ULs. The overall surface area of this limiter is $1.0 \mathrm{~m} \times 2.5 \mathrm{~m}$.

\subsection{Inboard Mid-plane Limiter (IML)}

Loss of confinement can lead to an H-L transition and the plasma would contract and touch the inboard wall [6]. Unlike any of the other limiters the IML will have to be accessed from its front; offering the biggest challenge among the limiters. It is proposed to access the IML from the equatorial port once the OML has been removed. This also means that the number of IMLs is the same as OMLs. To allow handling interface at the front of the limiter similar solution to that of ITER is sought [23], whereby the central part of the limiter, where the interface is located, would have to be shadowed by the PFCs.

The limiter would be attached directly to the vacuum vessel (VV) wall in between two inboard BB segments (Figure 4, left), again, to avoid having to split any inboard segments into two. The current concept envisages keys and load pads to the VV. 
The water coolant connection could be provided from the lower port direction. During operation the ferromagnetic forces are radial and pointing to the centre of the machine could be used to keep the limiter in place. The overall surface area of this limiter is $0.8 \mathrm{~m} \times 2.75 \mathrm{~m}$.

\section{First Wall and Limiter Shaping}

Unlike in ITER the DEMO FW will not be a limiter wall. During flat top operation the distance from the plasma will be $\sim 225 \mathrm{~mm}$ [24]. Despite that the FW panels have been shaped following a similar procedure to that of ITER [25-26] to optimise the heat load from charged particles. The shape of the BB FW front face has been set to match the flat top operation far scrape of layer (SOL) length.

As well as the FW the limiter surface has been shaped in a similar manner. However, each limiter surface has been optimised for the far SOL of the case against which they are intended to protect. Simulations have been run to check their heat load during flat top operation as well.

Initially, the limiter shape also included a $100 \mathrm{~mm}$ radius rounded edge just like the BB FW. Due to their protrusion the limiter edges see higher heat flux. The shape of these edges can be changed; however, a balance needs to be found that would not compromise the optimised shape but protect the edges too within the relatively short toroidal extent of the limiters.

The FW shape optimisation has started before limiters have been anticipated. With the limiters in place the wetted area of the FW is $\sim 8.4 \%$ of the total area, therefore shaping may not be necessary in the shaded area, also the tungsten armour of the FW could potentially be reduced (currently $\sim 2 \mathrm{~mm}$ ).

Understanding the impact on misalignments between blanket segments and limiters is crucial. The misalignments have various sources: manufacturing, assembly, operational differences, magnetic field lines etc. An initial study has been presented for multi-module segments as used until recently [27]. As the leading concepts are currently single module blanket segments a new study has been started which considers blanket segments as well as limiters in the study [28].

As much of the FW are is in shadow during flat top operation, future misalignment investigation can be focused on the wetted areas of the FW and the limiters.

Further work needs to be done to check the sensitivity of these shape to variance in the scrape of layer length, similar to misalignment studies. As a result of this exercise we may change the shape to a more resilient one even if that means going away from the optimised shape.

For the limiters the shape can be implemented in different ways: either by the monoblock heights or the cooling pipe shape or the most likely the combination of the two. A sensitivity study is under way to find out the impact of varying monoblock heights. So far, the study shows that tungsten thickness ranging from 12 to $25 \mathrm{~mm}$ can give adequate flexibility with regards to FW-shaping, especially at the rounded-edges of the limiters. Furthermore, the range of working front face thickness will allow the limiter to maintain its capability under erosion over long life time.

\section{Fabrication tests}

A development plan has been proposed in order to validate functional principles for DEMO Limiter and to acquire experience in all the processes required during the cycle of life for manufacturing and testing.

The industry infrastructure will have to be upgraded/created for fusion applications. It is one of the main objectives within this development plan to explore the market and the industrial capacities that are or will be needed to support fusion manufacturing and testing.

Currently, the mock-up fabrication tasks are on the way and they cover the PFC joining techniques. The main goal at this stage for WPBB is to validate the feasibility of the concept based on P91-steel layer as thermal break in order to decide if it is worth to pursue. P91 is a type of ferriticmartensitic steel micro alloyed with vanadium and niobium and with controlled nitrogen content.

Several filler metals (Orobraze ${ }^{\mathrm{TM}}$ 950, Orobraze ${ }^{\mathrm{TM}} 1025$, Pallabraze $^{\mathrm{TM}}$ 950, NBLM ${ }^{\mathrm{TM}}, \mathrm{H}-\mathrm{Bronze}^{\mathrm{TM}}$ ) are tested in order to develop a rational process of requirements and results with the possible PFC base materials (tungsten, P91, OFHC copper, $\mathrm{CuCrZr}$ ), defining matrix decision process that become lineal.

The two main requirements that drives this preselection are to guarantee the structural integrity during the service life of the component and to guarantee the proper brazing filler metal-base metal interaction.

\section{Summary}

The status of the EU DEMO limiter concept has been presented. Two design options have been explored for the limiter components, due to the maturity of the divertor concept the divertor-like concept has been chosen for the limiters too. The OML and UL are advanced while more work needs to be done for the IML and especially OLL, although solutions found for other limiters can be re-used. Work is still ongoing, both in CAD design as well as at the analysis front. Optimisation of the limiter cassette internals from thermo-hydraulic point of view as well as interfaces with the port plugs and remote maintenance equipment are the next priorities.

Analyses of charged particle heat flux based on the FW and limiter shaping show promising results that the limiters can protect the BB FW as well as have acceptable heat loads during steady-state operation. The limiter PFC shaping implementation has yet to be decided. The most likely solution for the PFC shaping is a combination of monoblock height and cooling pipe shape.

PFC joining fabrication and testing tasks for solid tungsten monoblocks are ongoing within the Breeding 
Blanket work package. R\&D for alternative PFC technologies are studied within the Divertor work package.

\section{Acknowledgements}

This work has been carried out within the framework of the EUROfusion Consortium and has received funding from the Euratom research and training programme 20142018 and 2019-2020 under grant agreement No 633053. The views and opinions expressed herein do not necessarily reflect those of the European Commission. To obtain further information on the data and models underlying this paper please contact publicationsmanager@ccfe.ac.uk.

\section{References}

[1] L.V. Boccaccini et al., Objectives and status of EUROfusion DEMO blanket studies, Fusion Engineering and Design 109-111 (2016), 1199-1206

[2] S. Ruck, F. Arbeiter, Thermohydraulics of ribroughened helium gas running cooling channels for first wall applications, Fusion Engineering and Design 109-111 (2016), 1035-1040

[3] P.A. Di Maio et al., On the optimization of the first wall of the DEMO water-cooled lithium lead outboard breeding blanket equatorial module, Fusion Engineering and Design 109-111 (2016), 335-341

[4] G. Federici et al., DEMO design activity in Europe: Progress and updates, Fusion Engineering and Design 136, (2018) 729-741

[5] C. Bachmann et al., Key Design Integration Issues addressed in the EU DEMO pre-concept design phase, ISFNT-14, O3-1.5

[6] F. Maviglia et al., Impact of Plasma Thermal Transients on the design of the EU DEMO, ISFNT$14, \mathrm{O} 1-2.1$

[7] T. Hirai et al., ITER tungsten divertor design development and qualification program, Fusion Engineering and Design 88, (2013) 1798-1801

[8] T. Hirai et al., ITER divertor materials and manufacturing challenges, Fusion Engineering and Design 125, (2017) 250-255

[9] E. Visca et al., Manufacturing and testing of ITERlike divertor plasma facing mock-ups for DEMO, Fusion Engineering and Design 136 (2018) 15931596

[10] J.H. You, et al., European DEMO divertor target: Operational requirements and material-design interface, Nuclear Materials and Energy, Vol. 9, Dec. 2016, Pages 171-176

[11] J.H. You et al., Progress in the initial design activities for the European DEMO divertor: Subproject "Cassette", Fusion Engineering and Design 124 (2017) 364-370
[12] T.R. Barrett et al., Designs and Technologies for plasma-facing wall protection in EU DEMO, Nuclear Fusion, (2019) In Press

[13] W. M. Rosenow, J. P. Hartnett and Y. I. Cho, Handbook of Heat Transfer, 3rd edition, McGrawHill, 1998.

[14] J. Boscary, Dimensional analysis of critical heat flux in subcooled water flow under one-side heating conditions for fusion application, Fusion Engineering and Design, 1998.

[15] A. Cardella et al., Design and manufacturing of the ITER limiter, Fusion Engineering and Design 61-62 (2002) 111-116

[16] V. Thompson et al., Analysis and design of the beryllium tiles for the JET ITER-like wall project, Fusion Engineering and Design 82 (2007) 17061712

[17] I. A. Maione et al., Update of Electromagnetic Loads on HCPB Breeding Blanked for DEMO 2017 Configuration, ISFNT-14, P1-096

[18] G. Mazzone et al., EuroFusion-DEMO Divertor Cassette Design and Integration, ISFNT-14, P1-029

[19] G. Mazzone et al, Choice of a low operating temperature for the DEMO EUROFER97 divertor cassette, Fusion Eng. Des. 124 (2017) 655-658

[20] T. Franke et al., The EU DEMO equatorial outboard limiter - design and port integration concept, ISFNT-14, P1-009

[21] R. Wenninger at al., The DEMO wall load challenge, Nuclear Fusion 57, (2017) 046002

[22] C. Vorpahl et al., Initial configuration studies of the upper vertical port of the European DEMO, Fusion Engineering and Design, (2019) In Press

[23] R. Mitteau, The design of the ITER first wall panels, Fusion Engineering and Design 88, (2013) 568-570

[24] T.R. Barrett et al., Progress in the engineering design and assessment of the European DEMO first wall and divertor plasma facing components, Fusion Engineering and Design 109-111, (2016) 917-924

[25] R. Mitteau, A shaped First Wall for ITER, Journal of Nuclear Materials 415, (2011) S969-S972

[26] P.C. Stangby, R. Mitteau, Analysis for shaping the ITER first wall, Journal of Nuclear Materials 390391, (2009) 963-966

[27] Z. Vizvary et al., DEMO First Wall misalignment study, Fusion Engineering and Design, 2019, In Press

[28] M. L. Richiusa et al., DEMO Single Module Segment Concept First Wall and limiter misalignment study by 3D field line tracing, ISFNT14, P1-040 